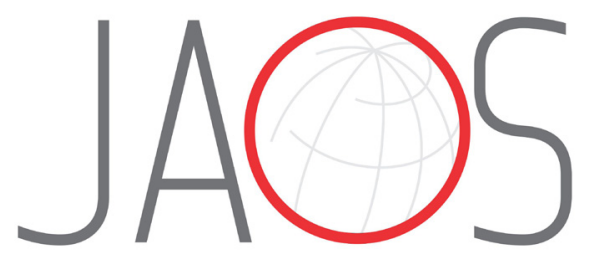
JOURNAL OF APPLIED ORAL SCIENCE

\title{
Preemptive use of etodolac on tooth sensitivity after in-office bleaching: a randomized clinical trial
}

\section{Abstract}

Savil Costa VAEZ 1

André Luís FARIA-E-SILVA ${ }^{1}$ Alessandro Dourado LOGUÉRCIO ${ }^{2}$

Micaelle Tenório Guedes FERNANDES ${ }^{1}$

Flávia Pardo Salata NAHSAN ${ }^{1}$
Submitted: September 29, 2016 Modification: July 8, 2017 Accepted: July 16, 2017

Corresponding address: Flávia Pardo Salata Nahsan Rua Cláudio Batista, s/n, Aracaju - SE 49060-100 - Brazil. Phone - Fax: +55 79 2105-1824 e-mail: flavia_odonto@hotmail.com
Purpose: This study determined the effectiveness of the preemptive administration of etodolac on risk and intensity of tooth sensitivity and the bleaching effect caused by in-office bleaching using 35\% hydrogen peroxide. Material and methods: Fifty patients were selected for this tripleblind, randomized, crossover, and placebo-controlled clinical trial. Etodolac (400 mg) or placebo was administrated in a single-dose 1 hour prior to the bleaching procedure. The whitening treatment with $35 \%$ hydrogen peroxide was carried out in two sessions with a 7-day interval. Tooth sensitivity was assessed before, during, and 24 hours after the procedure using the analog visual scale and the verbal rating scale. Color alteration was assessed by a bleach guide scale, 7 days after each session. Relative risk of sensitivity was calculated and adjusted by session, while overall risk was compared by the McNemar's test. Data on the sensitivity level of both scales and color shade were subjected to Friedman, Wilcoxon, and Mann-Whitney tests, respectively $(\alpha=0.05)$. Results: The preemptive administration of etodolac did not affect the risk of tooth sensitivity and the level of sensitivity reported, regardless of the time of evaluation and scale used. The sequence of treatment allocation did not affect bleaching effectiveness, while the second session resulted in additional color modification. The preemptive administration of etodolac in a single dose 1 hour prior to in-office tooth bleaching did not alter tooth color, and the risk and intensity of tooth sensitivity reported by patients. Conclusion: A single-dose preemptive administration of $400 \mathrm{mg}$ of etodolac did not affect either risk of tooth sensitivity or level of sensitivity reported by patients, during or after the in-office tooth bleaching procedure.

Keywords: Anti-inflammatory agents. Tooth bleaching. Dentin sensitivity. Drug prescriptions.
${ }^{1}$ Universidade Federal de Sergipe, Aracaju, SE, Brasil.

'Universidade Estadual de Ponta Grossa, Faculdade de Odontologia, Departamento de Odontologia Restauradora, Ponta Grossa, PR, Brasil. 


\section{Introduction}

Tooth whitening is a simple and non-invasive treatment commonly carried out to reestablish smile aesthetics. High success rates have been demonstrated for bleaching techniques applying 35\% hydrogen peroxide $(\mathrm{HP} 35 \%)^{2}$. Hydrogen peroxide $\left(\mathrm{H}_{2} \mathrm{O}_{2}\right)$-based bleaching agents at high concentrations (typically 15$38 \%$ ) are currently used for in-office techniques due to their high oxidizing ability ${ }^{12,25}$. However, the low molecular weight of $\mathrm{H}_{2} \mathrm{O}_{2}$ allows its penetration across the entire dentin tissue, reaching the pulp chamber and promoting damage of pulp stem cells, which is reported by patients as tooth sensitivity ${ }^{15,29}$.

Prior clinical trials have reported absolute risk of tooth sensitivity as high as $95 \%$ when highly concentrated $\mathrm{H}_{2} \mathrm{O}_{2}$ is used for in-office tooth bleaching $9,20,23,25$. Thus, the preemptive use of desensitizer agents ${ }^{3,30}$ or antiinflammatories $7,22,23,27$ has been proposed to reduce the risk of post-bleaching tooth sensitivity. Only the former significantly decreased tooth sensitivity; however, the application of desensitizers, when not incorporated into the bleaching gel, adds an extra step to the bleaching protocol, which is contrary to a clinician's need for simplification. On the other hand, the preemptive use of anti-inflammatories does not increase the number of steps in the bleaching protocol. Unfortunately, prior studies evaluating etoricoxib, ibuprofen, or dexamethasone were unable to demonstrate any beneficial effect on tooth sensitivity caused by tooth bleaching 7,13,22,23,28.

Etoricoxib and ibuprofen are grouped as class II drugs by the Biopharmaceutical Classification System (BCS), presenting low solubility and high permeability, which can hinder their absorption and create bioavailability mismatch during the bleaching procedure ${ }^{28}$. Moreover, anti-inflammatory drugs may have a specific action over inflammatory mediators (bradykinin) and the neurotransmitter (substance P) of tooth pain caused by dental bleaching ${ }^{6}$. Among non-steroid anti-inflammatory drugs (NSAIDs), etodolac demonstrated efficacy on the control of prostaglandins and bradykinins ${ }^{18}$. Nevertheless, there is no clinical evidence regarding the use of etodolac on the reduction of tooth sensitivity caused by in-office bleaching.

Thus, this study aimed to evaluate the effectiveness of the preemptive prescription of etodolac on risk of tooth sensitivity during and after in-office bleaching treatment. The first hypothesis evaluated was that etodolac would reduce both level and risk of tooth sensitivity when administrated in a single dose prior to in-office bleaching. The second hypothesis tested was whether the use of etodolac would reduce tooth sensitivity with no effect on tooth bleaching.

\section{Material and methods}

This clinical trial followed the CONSORT statements and was approved by the Scientific Review Committee and by the Committee for the Protection of Human Subjects of the local university (CAAE 37578714.4.0000.5546), and registered at clinicaltrials.gov under the number NCT02881619.

\section{Trial design}

This study was a randomized, triple-blind, placebocontrolled clinical trial with a crossover design. Patients included signed an informed consent form and were submitted to two in-office bleaching sessions receiving placebo (control) or etodolac prior to the bleaching procedure, while different treatments were allocated for each session.

A one-week interval ("washout") in-between sessions was established. The study was conducted at the clinic of the School of Dentistry of the local university from November 2014 to July 2015.

\section{Participants}

Patients from 18 to 35 years old with good oral health were included in this clinical trial. From the patients who received placebo/etodolac, 6 were men and 19 were women, and for etodolac/placebo, 12 were men and 13 were women; the average age was 23 years; 64\% were women.

Patients with any of the six upper anterior teeth with caries, restoration, severe discoloration (e.g., stains caused by tetracycline), enamel hypoplasia, gingival recession, dentin exposure, pulpitis, or endodontics were excluded, as well as smokers. Participants submitted to previous bleaching procedures, with prior tooth sensitivity, known allergy to any component of the medication used in the study, and pregnant or breastfeeding women were also excluded. An air stream was applied to teeth to verify the presence or absence of sensitivity (none or zero). Only patients presenting all six upper anterior teeth with shade 
mismatch of 2.5 M2 (Vita Bleach guide 3D-Master scale, Vita-Zahnfabrik, Bad Sackingen, Germany) - A3.5 Vita Classical equivalence - or darker were included.

\section{Sample size calculation}

The sample calculation was based on the primary binary outcome (sensitivity risk 24 hours after the procedure) for superiority trial. The power of the test was set at $80 \%$, considering a type I error of 0.05 and risk of tooth sensitivity of $90 \%$, based on a prior study using a similar bleaching agent ${ }^{23}$; moreover, a reduction around $30 \%$ was expected after treatment. The calculation resulted in fifty patients.

\section{Randomization}

A randomized list was computer-generated by a person not involved in intervention or evaluation. Participants were defined as blocks in the randomization process, in which the sequence of treatment (placebo or etodolac) was randomly set for each block by computer-generated tables (www.sealedenvelope. com). The sequence was inserted into sealed envelopes numbered from 1 to 50 that were opened by the operator only at the moment of intervention. Patients were numbered according to the sequence of enrollment. Neither the participant nor the operator knew the group allocation, determining a blinded protocol.

\section{Baseline evaluation}

Prior to the bleaching procedure, teeth were cleaned using rubber cups associated with pumice and water. The shades of upper incisors and canine teeth $(13,12,11,21,22$, and 23) were assessed on a baseline using the bleach guide scale. Color was analyzed by two previously calibrated evaluators. Both evaluators presented superior color matching competency according to the ISO (International Organization for Standardization)/TR 28642:2011. Shade tabs selected were converted to scores ranging from 1 (whiter shade - OM1) to 15 (darker shade 5M3).

Considering a possible effect of dental anxiety on the sensitivity reported by patients, the Corah's Dental Anxiety Scale was used to determine the level of anxiety of each patient related to the procedure ${ }^{17}$. Each answer to the survey instrument was scored on a scale from 1 to 5 (four questions) and the sum of scores was used to determine the level of anxiety: low was under 12, moderate was between 12 and 14, and high was over 14 .

\section{Intervention}

One hour before each bleaching session and right after the prophylaxis, patients received a capsule containing $400 \mathrm{mg}$ of NAISE etodolac (Flancox ${ }^{\mathrm{TM}}$, Apsen Farmaceutica S/A) or 400 mg of placebo (inert content) according to randomization. Capsules had the same appearance and were manufactured by a person not involved in intervention or evaluation. They were placed into two bottles identified by letters according to the treatment. Neither the operators responsible for intervention and evaluation nor the patients knew the content of each capsule.

The color evaluation was verified, and the lightcured resin dam was applied (Top Dam, FGM, Joinville, SC, Brazil) and polymerized (Radii-cal, SDI, Bayswater, Australia) on the gingival tissue corresponding to the teeth to be bleached. A 35\% hydrogen peroxide-based bleaching agent (Whiteness HP Blue, FGM, Joinville, SC, Brazil) was mixed and applied to the buccal surface of teeth for 40 minutes. After that, the bleaching agent was removed. A second session was carried out after a week following the same procedures. At this time, the patient received a single-dose capsule containing etodolac or placebo (different from the ones received at the first session), one hour before the procedure. During the bleaching treatment, patients were advised not to ingest colored food and beverages.

\section{Evaluations}

Tooth sensitivity reported by patients was assessed using a visual analog scale (VAS) and a verbal rating scale (VRS). The VAS consisted of a $10-\mathrm{cm}$ long scale ranging from green (absence of pain) to red (unbearable pain). Patients set their level of sensitivity by pointing to the color corresponding to the pain level, while the distance from this point to the green border was recorded. For the VRS, patients reported their level of sensitivity based on scores: $0=$ none; $1=$ mild; $2=$ moderate $; 3=$ considerable ; and $4=$ severe. Tooth sensitivity was assessed during bleaching, immediately after removing the bleaching agent, and after 24 hours. For this last assessment, only the VRS was used due to the difficulty of patients to fill the VAS at home. One week after each session, tooth color was evaluated again using the same procedure described previously. 


\section{Statistical analysis}

Demographic data from patients were analyzed to determine age, gender, and anxiety level for each allocation sequence. Comparisons among allocation sequences were performed by Mann-Whitney (age), Fisher's exact (gender), and chi-square (anxiety level) tests.

Based on the presence of any tooth sensitivity (VRS scores different from 0 ), the absolute risk, odds ratio, and relative risk were calculated regarding the treatments for each moment of bleaching evaluation/ session, as well as the confidence intervals (95\%). For each moment, differences on presence/absence ratios were analyzed by Fisher's exact test. For the overall risk related to each treatment, odds ratio was adjusted to the independent variable of "session of bleaching" using Mantel-Haenszel statistics. The homogeneity of odds ratios was analyzed by Breslow-Day and Tarone's tests. Next, the estimated odds ratio was converted to relative risk and the overall presence/absence ratios were analyzed by the McNemar's test, considering the study design (crossover).

For the VRS, data from scores observed at each moment of bleaching evaluation/session were submitted to the Mann-Whitney rank sum test. Despite the measurement of tooth sensitivity using the VAS, which provided a continuous outcome, data assessed with this scale did not show normal distribution (Shapiro-Wilk's test). Thus, data from the VAS were also analyzed by the Mann-Whitney rank sum test, performing one test per time of evaluation.

For color evaluation, comparisons among sequences of treatment were performed using the Mann-Whitney rank sum test. Friedman test followed by Dunn's post hoc test were used to analyze the difference between the moments of evaluation for each sequence of treatment. All statistical analyses were performed adjusting the initial significance level $(a=0.05)$ by the Bonferroni correction.

\section{Results}

Figure 1 shows the flow chart of patients assessed for eligibility, who were included in the study and analyzed. Table 1 shows the demographic characteristics of patients allocated for each sequence of treatment. Regarding anxiety, $88 \%$ of patients had a low level and only $2 \%$ had a high level $(p=0.236)$. There was no difference among the sequences of treatment for any demographic characteristic analyzed (age: $p=0.089$ and gender: $p=0.140$ ).

Table 2 shows the results of tooth sensitivity risk. The treatment that patients received prior to the

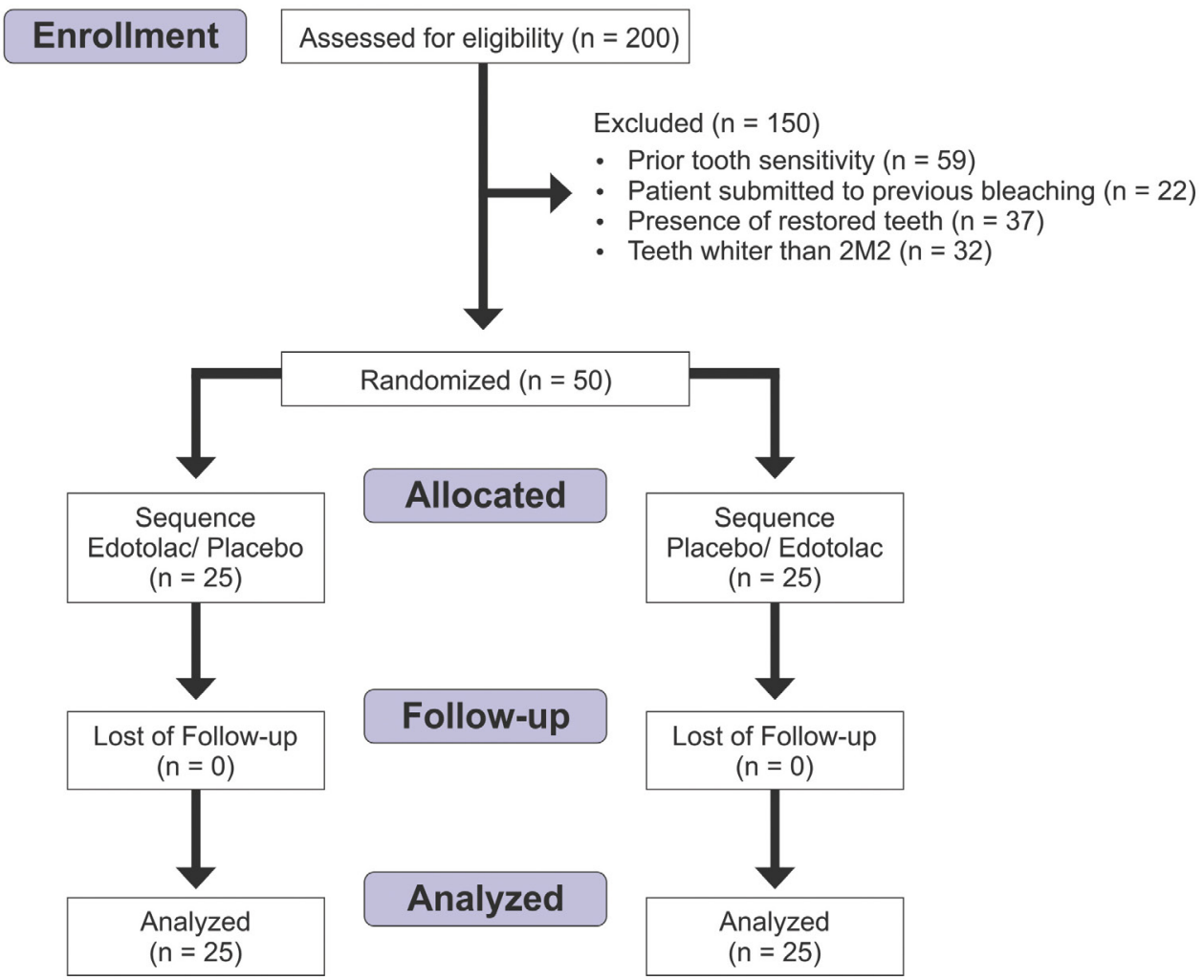

Figure 1- Flow chart of patients 
Table 1- Profile of patients included in the study allocated to each sequence of treatment

\begin{tabular}{|c|c|c|c|}
\hline Age (years) & & $\begin{array}{c}\text { Median } \\
\left(1^{\text {st }} / 3^{\text {rd }} \text { quartiles }\right)\end{array}$ & p-value \\
\hline Total & & $23.0(21.0 / 26.0)$ & \\
\hline Placebo/Edotolac & & $23.8(21.0 / 25.5)$ & $p^{*}=0.089$ \\
\hline Edotolac/Placebo & & $25.0(22.0 / 28.0)$ & \\
\hline \multirow[t]{3}{*}{ Gender Total } & & n (\%) & \\
\hline & Male & $18(36.0 \%)$ & \\
\hline & Female & $32(64.0 \%)$ & \\
\hline \multirow[t]{3}{*}{ Placebo/Edotolac } & & & $p^{* *}=0.140$ \\
\hline & Male & $6(24.0 \%)$ & \\
\hline & Female & $19(76.0 \%)$ & \\
\hline \multicolumn{4}{|l|}{ Edotolac/Placebo } \\
\hline & Male & $12(52.0 \%)$ & \\
\hline & Female & $13(48.0 \%)$ & \\
\hline \multirow[t]{4}{*}{ Level of anxiety Total } & & n (\%) & \\
\hline & Low anxiety & $44(88.0 \%)$ & \\
\hline & Moderate anxiety & $5(10.0 \%)$ & \\
\hline & High anxiety & $1(2.0 \%)$ & \\
\hline \multirow[t]{4}{*}{ Placebo/Edotolac } & & & $\mathrm{p}^{* * *}=0.236$ \\
\hline & Low anxiety & $21(84.0 \%)$ & \\
\hline & Moderate anxiety & $4(16.0 \%)$ & \\
\hline & High anxiety & $0(0.0 \%)$ & \\
\hline \multicolumn{4}{|l|}{ Edotolac/Placebo } \\
\hline & Low anxiety & $23(92.0 \%)$ & \\
\hline & Moderate anxiety & $1(4.0 \%)$ & \\
\hline & High anxiety & $1(4.0 \%)$ & \\
\hline
\end{tabular}

* Mann-Whitney rank sum test; ${ }^{* *}$ Fisher Exact test; ${ }^{* *}$ Chi-square test.

Table 2- Results of risk of tooth sensitivity observed for each treatment

\begin{tabular}{|c|c|c|c|c|c|c|c|}
\hline Session & Moment of evaluation & During & & $\begin{array}{l}\text { Immediately } \\
\text { after }\end{array}$ & & $24 \mathrm{~h}$ after & \\
\hline & Treatment & Edotolac & Placebo & Edotolac & Placebo & Edotolac & Placebo \\
\hline \multirow[t]{4}{*}{$1^{\text {st }}$ session } & Presence of sensitivity (yes/no) & $(10 / 15)$ & $(11 / 14)$ & $(9 / 16)$ & $(10 / 15)$ & $(3 / 22)$ & $(2 / 23)$ \\
\hline & Odds ratio $(95 \% \mathrm{Cl})$ & $\begin{array}{c}0.52 \\
(0.17-1.61)\end{array}$ & & $\begin{array}{c}0.84 \\
(0.27-2.65)\end{array}$ & & $\begin{array}{c}1.57 \\
(0.24-10.30)\end{array}$ & \\
\hline & Relative risk $(95 \% \mathrm{Cl})$ & $\begin{array}{c}0.71 \\
(0.40-1.29)\end{array}$ & & $\begin{array}{c}0.90 \\
(0.44-1.83)\end{array}$ & & $\begin{array}{c}1.50 \\
(0.27-8.22)\end{array}$ & \\
\hline & $p$-value ${ }^{*}$ & 0.396 & & 1.000 & & 1.000 & \\
\hline \multirow[t]{4}{*}{$2^{\text {nd }}$ session } & Presence of sensitivity (yes/no) & $(8 / 17)$ & $(5 / 20)$ & $(6 / 19)$ & $(4 / 21)$ & $(1 / 24)$ & $(1 / 24)$ \\
\hline & Odds ratio $(95 \% \mathrm{Cl})$ & $\begin{array}{c}1.88 \\
(0.52-6.85)\end{array}$ & & $\begin{array}{c}1.66 \\
(0.41-6.79)\end{array}$ & & $\begin{array}{c}1.00 \\
(0.59-16.93)\end{array}$ & \\
\hline & Relative risk $(95 \% \mathrm{Cl})$ & $\begin{array}{c}1.60 \\
(0.61-4.22)\end{array}$ & & $\begin{array}{c}1.50 \\
(0.48-4.68)\end{array}$ & & $\begin{array}{c}1.00 \\
(0.06-15.12)\end{array}$ & \\
\hline & $p$-value* & 0.520 & & 0.725 & & 1.000 & \\
\hline \multirow[t]{3}{*}{ Average } & Odds ratio $(95 \% \mathrm{Cl})^{\star *}$ & $\begin{array}{c}0.92 \\
(0.40-2.09)\end{array}$ & & $\begin{array}{c}1.11 \\
(0.46-2.67)\end{array}$ & & $\begin{array}{c}1.37 \\
(0.29-6.51)\end{array}$ & \\
\hline & Relative risk ${ }^{\star * *}$ & 0.95 & & 1.08 & & 1.34 & \\
\hline & $p$-value $e^{* * *}$ & 1.000 & & 1.000 & & 0.683 & \\
\hline
\end{tabular}

* Fisher exact test; ** Mantel-Haenszel common odds ratio estimate; ${ }^{* * \star}$ Based on odds ratio estimated; ****McNemar's test. The cut-off value of type I error $(\alpha=0.0056)$ was adjusted by Bonferroni correction. 
bleaching procedure did not affect the risk to sensitivity at any of the moments of evaluation (during and immediately after: $p=1.0$; after 24 hours: $p=0.683$ ).
Figure 2 shows the results for level of sensitivity assessed by the VRS. Treatment did not affect level of sensitivity, regardless of the moment of evaluation.

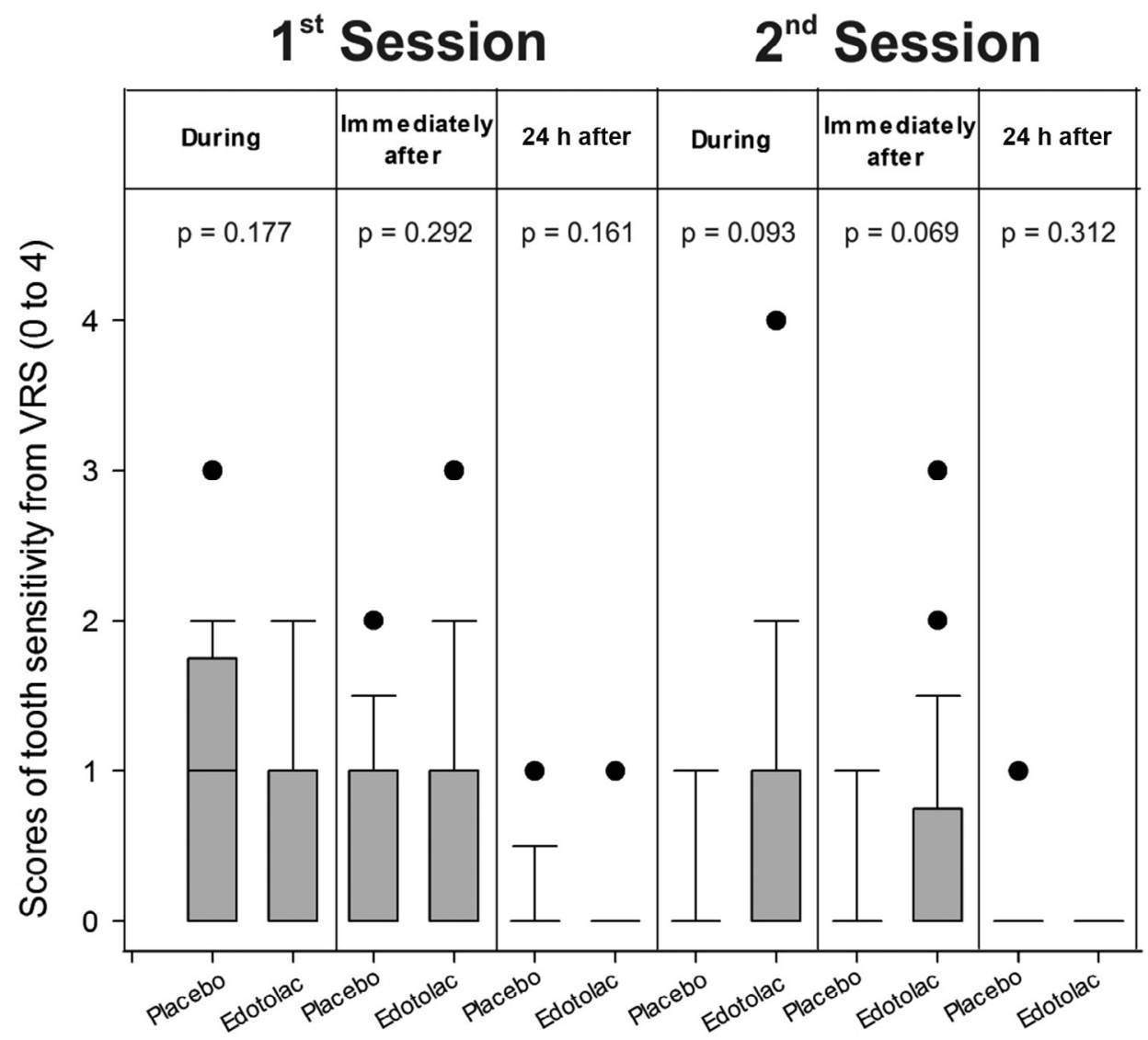

Figure 2 - Scores of tooth sensitivity from the VRS (0 to 4)

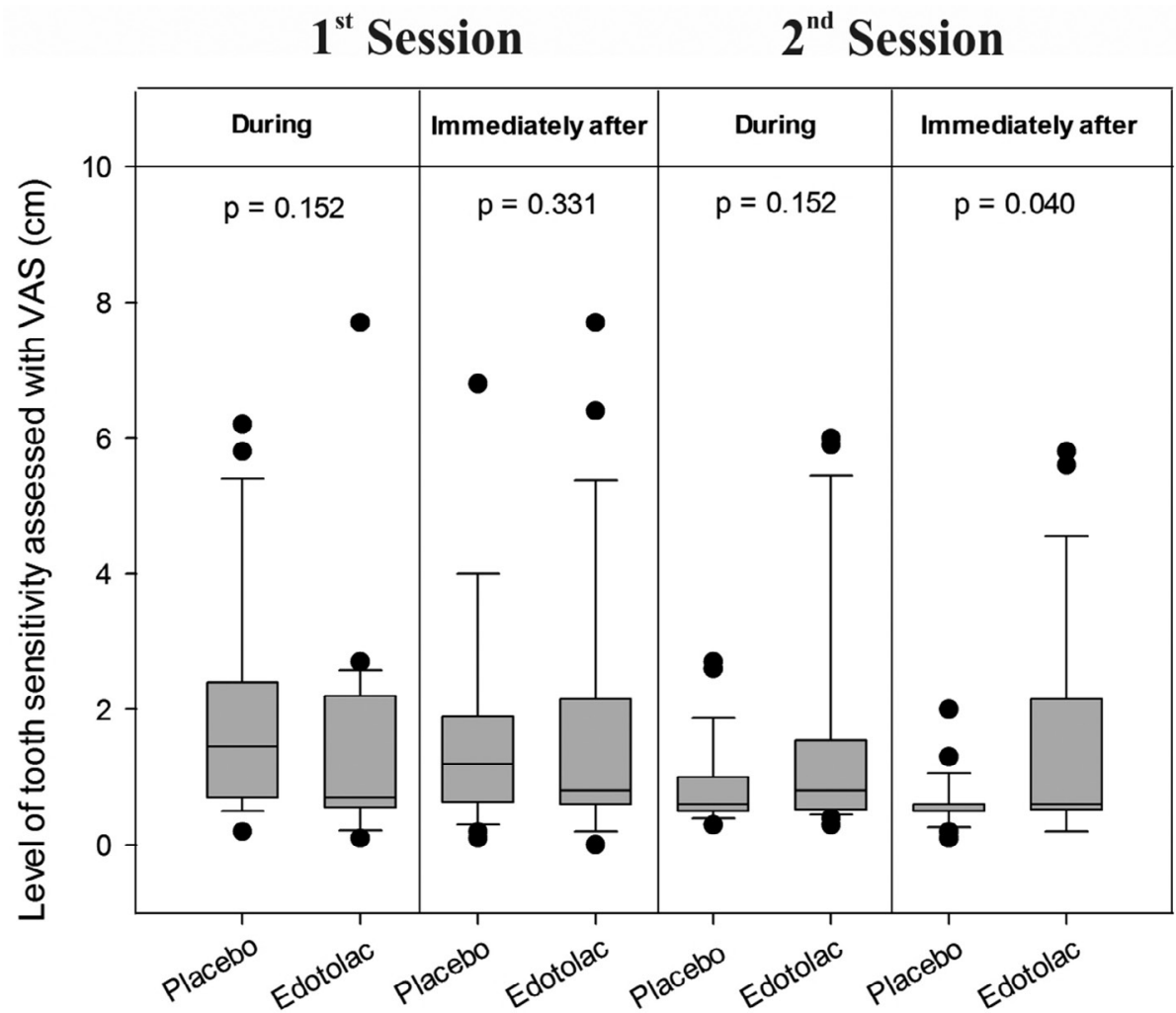

Figure 3- Level of tooth sensitivity assessed with VAS (cm) 


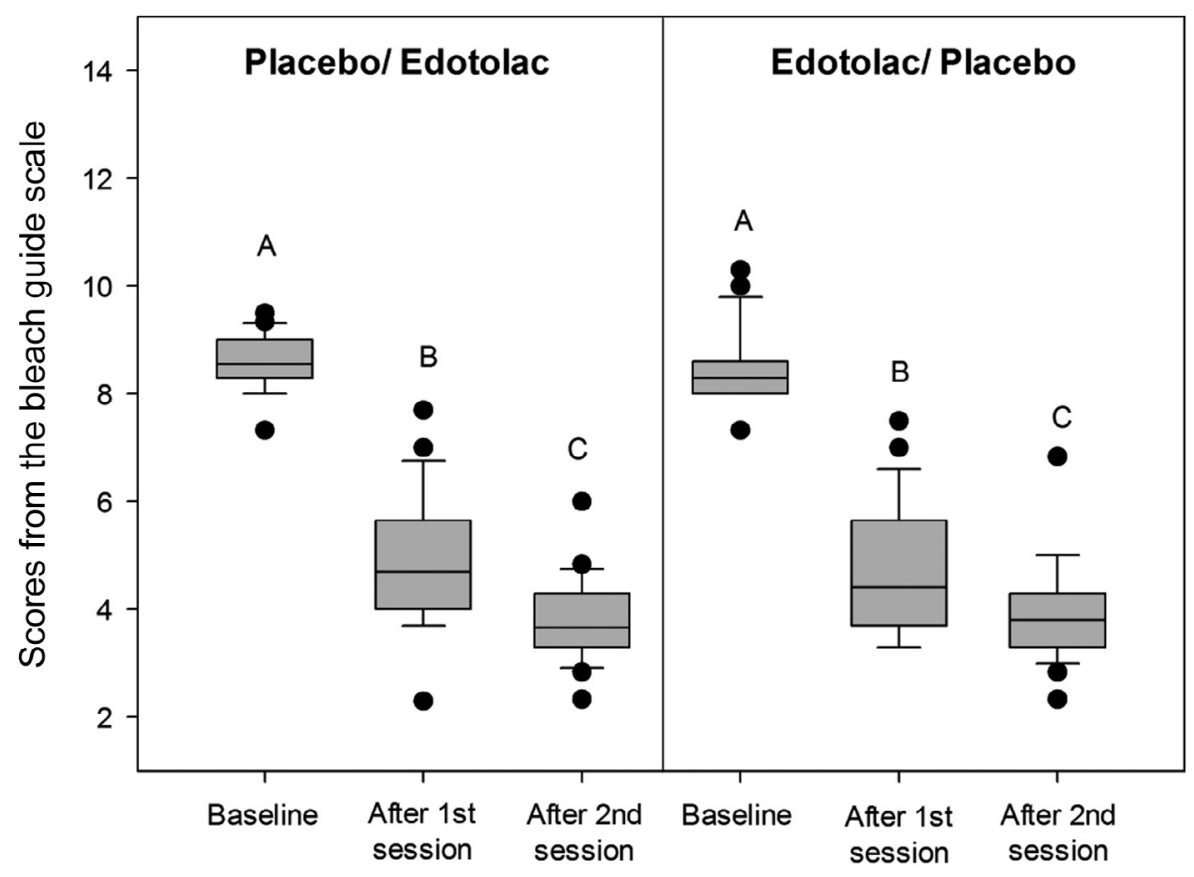

Figure 4- Scores from the bleach guide scale

Similar results were observed when the VAS was used (Figure 3).

In regards to bleaching effectiveness, the bleaching procedure carried out in this study was able to significantly reduce the shade scores from the bleach guide, while the second session resulted in additional bleaching effect (Figure 4). The sequence of treatment did not affect bleaching effectiveness.

\section{Discussion}

Tooth bleaching performed by patients at home using low-concentration peroxides has been reported as the first-choice technique for vital bleaching, and probably the most widely used one ${ }^{5,11}$. However, procedures using high-concentration bleaching agents applied in office by clinicians remain an important protocol used to bleach discolored teeth for specific indications of tooth bleaching. This protocol is separate and intended to aid patients who cannot adapt to the use of home-based bleaching trays and who have contraindications related to the gastric system, because it reduces the risk of gel intake ${ }^{14}$, and to control the risk factor for developing gingival irritation ${ }^{10}$. In-office bleaching is also indicated to patients requiring faster results, while this technique can be combined with at-home bleaching ${ }^{26}$.

In-office techniques have demonstrated high bleaching effectiveness using high-concentration hydrogen peroxides ${ }^{26}$. However, high-concentrated bleaching agents also result in increased tooth sensitivity reported by patients during and up to 24 hours after the bleaching procedure, which is the main adverse effect related to in-office tooth bleaching, 9,20,21. Even though tooth sensitivity is related to the inflammatory process of pulp tissue ${ }^{20}$, the findings of this study showed that the preemptive use of etodolac in a single dose did not affect the risk and level of tooth sensitivity caused by in-office bleaching. As expected, preemptive administration of etodolac also did not affect the bleaching results. Thus, the first hypothesis of the study was rejected and the second one was accepted.

Unlike the tooth sensitivity typically reported by patients presenting teeth with dentin exposure, which relates mainly to thermal stimuli, bleached teeth can hurt even in the absence of any stimulus, showing that the pain mechanism related to peroxides is different from other types of tooth pain ${ }^{20}$. Moreover, the sensitivity caused by tooth bleaching tends to increase within a few hours following the bleaching procedure, when most patients described the pain as a "twinge" or "shock-like." It has been demonstrated that the oxidizing agents used during the bleaching procedure cause a reduction on metabolism, viability, and cell proliferation ${ }^{20}$, allowing to increase the expression of inflammatory mediators, such as substance $P$ and bradykinin, which is a vasoactive peptide released by nerves resulting in a neurogenic inflammation ${ }^{6}$. Thus, a 
preemptive administration of anti-inflammatories could be a reasonable approach to reduce tooth sensitivity associated with bleaching procedures. However, in this study, sensitivity was not lower during and 24 hours after the bleaching procedure. Unfortunately, the peak of tooth sensitivity was not measured in this study, even though this outcome could allow to assess a possible effect of etodolac on sensitivity following the end of the bleaching procedure.

A prior study demonstrated that etodolac presents higher effectiveness on bradykinin inhibition than other drugs commonly used to control the inflammatory process, motivating its use in this study ${ }^{18}$. Regarding pharmacokinetics, etodolac reaches its maximal plasma concentration around 1 to 2 hours after its administration ${ }^{4}$. Thus, it was expected that maximal plasma concentration would be reached during the bleaching procedure. Unfortunately, no effect of the preemptive administration of etodolac on the reported tooth sensitivity was observed at this time of evaluation for both sessions. Despite its demonstrated efficacy on inflammation control, the analgesic effect of etodolac increases when its administration is repeated, whereas a single dose prior to the bleaching procedure seems insufficient to prevent tooth sensitivity ${ }^{19}$.

Another important observation regarding pharmacokinetics is that a crossover design was used in this study with a 1-week interval period. Considering that the half-life of etodolac after oral administration is around 13 hours ${ }^{16}$, it is expected to find no residual effect after 1-week. Moreover, the crossover design avoids bias related to pain thresholds of patients ${ }^{1}$.

Participants included in this study were predominantly young females presenting low level of anxiety. All these demographics characteristics of the studied population might be associated with differences in pain thresholds. Higher tooth sensory threshold has been demonstrated in males due to differences in crown diameters of teeth and underlying mechanisms such as neurological differences or behavior aspects ${ }^{8}$. Regarding the age of participants, a recent review did not find any relation between age and the risk to or level of tooth sensitivity ${ }^{27}$. However, it is important to emphasize that most participants from trials included in that review were under 30 years old ${ }^{27}$. Another important demographic aspect assessed in this study was the participants' level of dental anxiety prior to bleaching procedures. It has been demonstrated that dental anxiety is a strong predictor of pain and that anxious participants are prone to develop painful responses ${ }^{24}$. In this research, almost $90 \%$ of participants presented low anxiety prior to bleaching procedures, which can be justified by the low invasive aspect of intervention, despite the patients' concern about tooth sensitivity. In fact, despite $66 \%$ of participants reporting various level of tooth sensitivity (high risk), the actual level of sensitivity reported was low (medians below moderate at VRS, and means lower than 2 at VAS).

In addition to the evaluation of tooth sensitivity, we also assessed color alteration promoted by bleaching procedures. The data analysis of color evaluation used the sequence of allocation instead of the treatment (placebo or etodolac). If we had used treatment, different participants between the first and second sessions would be compared for the same treatment, which would have impaired correct color changes assessment. Moreover, the main aim of color evaluation was to show the effectiveness of the bleaching technique used.

In our study, the protocol that was carried out resulted in significant bleaching effect, while an additional color alteration was achieved at the second session. In addition, the last color evaluation was performed a week after the last bleaching session of this study, while longer times may be required for color stabilization ${ }^{21}$. However, a shorter time was used because the tooth sensitivity reported by patients was the main outcome of this trial. It has been demonstrated that two sessions of in-office tooth bleaching results in a mean change of 5.3 ( \pm 2.8) units on shade guides ${ }^{26}$, which is similar to the average color change achieved in this research. Factors such as patient's age and color at baseline have been strongly associated with bleaching effectiveness, while young patients and darker teeth show more pronounced color changes ${ }^{27}$. We found no difference in baseline regarding these parameters between the sequence of allocation, while the inclusion of young participants presenting all teeth darker than 2.5 M2 (score 7) favored obtaining significant color bleaching.

In conclusion, the preemptive administration of etodolac in a single dose 1 hour prior to the bleaching procedure was unable to reduce both risk and level of sensitivity caused by in-office bleaching. A limitation of this study was that the preemptive treatment was administrated only for a young population (average age of 23 years), with prevalence of female patients, 
while different results can be observed for other demographic profiles ${ }^{27}$.

\section{Conclusions}

The preemptive administration of a single dose of etodolac previously to the two bleaching sessions with $35 \%$ hydrogen peroxide did not affect tooth color change, risk of sensitivity and level of pain reported by the patients (during the sessions, immediately after, and $24 \mathrm{~h}$ after sessions).

\section{Acknowledgments}

The authors would like to acknowledge the assistance provided by Lucas Simon Fülber, Márcia Louise Sobral, Ilzes Marianne Borges de Souza, and Natalia Andrade de Resende Pereira.

\section{References}

1- Bartley EJ, Fillingim RB. Sex differences in pain: a brief review of clinical and experimental findings. $\mathrm{Br}$ J Anaesth. 2013;111(1):52-8.

2- Bernardon JK, Ferrari P, Baratieri LN, Rauber GB. Comparison of treatment time versus patient satisfaction in at-home and in-office tooth bleaching therapy. J Prosthet Dent. 2015;114(6):826-30.

3- Bonafé E, Loguercio AD, Reis A, Kossatz S. Effectiveness of a desensitizing agent before in-office tooth bleaching in restored teeth. Clin Oral Investig. 2014;18(3):839-45.

4- Brocks DR, Jamali F. Etodolac clinical pharmacokinetics. Clin Pharmacokinet. 1994;26(4):259-74.

5- Brunton PA, Burke FJ, Sharif MO, Creanor S, Hosey MT, Mannocci F, et al. Contemporary dental practice in the UK in 2008: aspects of direct restorations, endodontics and bleaching. Br Dent J. 2012;212(2):63-7. 6- Caviedes-Bucheli J, Ariza-García G, Restrepo-Méndez S, Ríos-Osorio $\mathrm{N}$, Lombana N, Muñoz HR. The effect of tooth bleaching on substance $P$ expression in human dental pulp. J Endod. 2008;34(12):1462-5.

7- Charakorn P, Cabanilla LL, Wagner WC, Foong WC, Shaheen J, Pregitzer $\mathrm{R}$, et al. The effect of preoperative ibuprofen on tooth sensitivity caused by in-office bleaching. Oper Dent. 2009;34(2):131-5. 8- Chunhacheevachaloke $E$, Ajcharanukul O. Effects of conducting media and gender on an electric pulp test. Int Endod J. 2016;49(3):237-44. 9- Correa A, Santana T, Nahsan F, Loguercio A, Faria-e-Silva A. The impact of a customized tray on in-office bleaching tooth sensitivity: a randomized clinical trial. Oper Dent. 2016;41(1):15-22.

10- Dahl JE, Pallesen U. Tooth bleaching: a critical review of the biological aspects. Crit Rev Oral Biol Med. 2003;14(4):292-304.

11- Demarco FF, Conde MC, Ely C, Torre EN, Costa JR, Fernández MR, et al. Preferences on vital and nonvital tooth bleaching: a survey among dentists from a city of Southern Brazil. Braz Dent J. 2013;24(5):527-31. 12- Eimar H, Siciliano R, Abdallah MN, Nader SA, Amin WM, Martinez $\mathrm{PP}$, et al. Hydrogen peroxide whitens teeth by oxidizing the organic structure. J Dent. 2012;40 (Suppl 2):e25-33.
13- Faria-e-Silva AL, Nahsan FP, Fernandes MT, Martins-Filho PR. Effect of preventive use of nonsteroidal anti-inflammatory drugs on sensitivity after dental bleaching. J Am Dent Assoc. 2015;146(2):87-93.e1. 14- Goldberg M, Grootveld M, Lynch E. Undesirable and adverse effects of tooth-whitening products: a review. Clin Oral Investig. 2010;14(1):1-10.

15- He B, Huang S, Zhang C, Jing J, Hao Y, Xiao L, et al. Mineral densities and elemental content in different layers of healthy human enamel with varying teeth age. Arch Oral Biol. 2011;56(10):997-1004. 16- Honda K, Nakamura A, Morino A, Nomura A. Studies on the metabolic fate of etodolac (1) absorption, distribution and excretion in mice, rats, and dogs after single administration. Iyakuhin Kenkyu. $1991 ; 22: 109-23$.

17- Hu LW, Gorenstein C, Fuentes D. Portuguese version of Corah's Dental Anxiety Scale: transcultural adaptation and reliability analysis. Depress Anxiety. 2007;24(7):467-71.

18- Inoue $\mathrm{K}$, Motonaga A, Dainaka J, Nishimura T, Hashii H, Yamate $\mathrm{K}$, et al. Effect of etodolac on prostaglandin E2 biosynthesis, active oxygen generation and bradykinin formation. Prostaglandins Leukot Essent Fatty Acids. 1994;51(6):457-62.

19- Ito S, Tajima K, Nogawa M, Inoue N, Kyoi T, Takahashi Y, et al. Etodolac, a cyclooxygenase- 2 inhibitor, attenuates paclitaxel-induced peripheral neuropathy in a mouse model of mechanical allodynia. J Pharmacol Exp Ther. 2012;342(1):53-60.

20- Kose C, Calixto AL, Bauer J, Reis A, Loguercio AD. Comparison of the effects of in-office bleaching times on whitening and tooth sensitivity: a single blind, randomized clinical trial. Oper Dent. 2016;41(2):138-45. 21- Markowitz K. Pretty painful: why does tooth bleaching hurt? Med Hypotheses. 2010;74(5):835-40.

22- Paula E, Kossatz S, Fernandes D, Loguercio A, Reis A. The effect of perioperative ibuprofen use on tooth sensitivity caused by in-office bleaching. Oper Dent. 2013;38(6):601-8.

23- Paula EA, Loguercio AD, Fernandes D, Kossatz S, Reis A. Perioperative use of an anti-inflammatory drug on tooth sensitivity caused by in-office bleaching: a randomized, triple-blind clinical trial. Clin Oral Investig. 2013;17(9):2091-7.

24- Racek AJ, Hu X, Nascimento TD, Bender MC, Khatib L, Chiego D, et al. Different brain responses to pain and its expectation in the dental chair. J Dent Res. 2015;94(7):998-1003.

25- Reis A, Tay L, Herrera D, Kossatz S, Loguercio A. Clinical effects of prolonged application time of an in-office bleaching gel. Oper Dent. 2011;36(6):590-6.

26- Rezende M, Ferri L, Kossatz S, Loguercio AD, Reis A. Combined bleaching technique using low and high hydrogen peroxide in-office bleaching gel. Oper Dent. 2016;41(4):388-96.

27- Rezende M, Loguercio AD, Kossatz S, Reis A. Predictive factors on the efficacy and risk/intensity of tooth sensitivity of dental bleaching: a multi regression and logistic analysis. J Dent. 2016;45:1-6.

28- Rivera-Leyva J, García-Flores M, Valladares-Méndez A, OrozcoCastellanos $L$, Martínez-Alfaro M. Comparative studies on the dissolution profiles of oral ibuprofen suspension and commercial tablets using biopharmaceutical classification system criteria. Indian J Pharm Sci. 2012;74(4):312-8.

29- Shackelford RE, Kaufmann WK, Paules RS. Oxidative stress and cell cycle checkpoint function. Free Radic Biol Med. 2000;28(9):1387-404. 30- Tay LY, Kose C, Loguercio AD, Reis A. Assessing the effect of a desensitizing agent used before in-office tooth bleaching. J Am Dent Assoc. 2009;140(10):1245-51. 